\title{
Pricing correlation options: from the P. Carr and D. Madan approach to the new method based on the Fourier transform ${ }^{1}$
}

\author{
Arkadiusz Orzechowski ${ }^{2}$
}

\begin{abstract}
Pricing of options plays an important role in the financial industry. Investors knowing how to price derivative contracts quickly and accurately can beat the market. On the other hand market participants constructing their investment strategies with the use of options based on techniques that do not assure the highest computational speed and efficiency are doomed to failure. The aim of the article is to extend the existing methodology of pricing correlation options based on the Fourier transform. The article starts with a presentation of Carr and Madan's concept (Carr \& Madan, 1999). Then other methods of pricing options with the use of the Fourier transform are summarized. Finally, a new approach to pricing derivative contracts is derived and then applied to the correlation options.
\end{abstract}

Keywords: Fourier transform, pricing of options, correlation options.

JEL Codes: G13, C02.

\section{Introduction}

The rapid development of option markets worldwide in recent years is accompanied by a rise in trading activities of investors and a development of new, highly sophisticated, financial products. One direct consequence of such a trend is the possibility for buyers and sellers of the derivatives to more efficiently speculate on the movements of interest and exchange rates, commodity and equity prices, as well as credit ratings of the issuers of bonds. As the capacity for speculation using derivatives is growing, increased demand for new instruments allowing to hedge against different aspects of risks appears. Options, along with forwards and futures, seem to be the best tools that can be used to protect market participants against potential capital losses. One of the most interesting type of options used for managing risks are exotic options where pay-offs depend on

\footnotetext{
${ }^{1}$ Article received 15 February 2017, accepted 22 January 2018.

${ }^{2}$ Warsaw School of Economics, Collegium of Socio-Economics, Department of Banking, ul. Wiśniowa 41, 02-520 Warszawa, aorzec@sgh.waw.pl.
} 
the correlation between asset prices, e.g. exchange options, quotient options, correlation options. Some of the contracts, e.g. spread options, are traded on exchanges (NYMEX, NYCE, CBOT), others, like quotient and product options, can be bought or sold in the OTC market (Zhu, 2000).

The aim of the article is to extend the analysis of the Carr and Madan method (Carr \& Madan, 1999) for pricing correlation options in the Black and Scholes framework (Black \& Scholes, 1973). The article consists of several sections. At the beginning the martingale method is applied to the valuation of analyzed derivatives. Next, the method is modified to the form proposed by Carr and Madan (1999). Finally, alternative methods of pricing correlation options, which are based on the Fourier transform, are presented and a new model for pricing of this type of derivatives is developed.

\section{Pricing correlation options using the martingale method}

One of the most popular methods of pricing correlation options is based on the risk-neural concept (method referred to as BS-M) (Black \& Scholes, 1973). The method is based on the assumption that the price of the underlying instrument follows geometric Brownian motion. In consequence the natural logarithm of market value of the underlying instrument has normal distribution. The joint probability density function of the two-dimensional variable that has to be analyzed for the correlation options can be described with the use of an elegant formula as follows:

$$
\begin{aligned}
& \tilde{q}\left(S_{1 T}, S_{2 T}\right)=\frac{1}{2 \pi \tau \sigma_{1} \sigma_{2} \sqrt{1-\rho^{2}}} \times \\
& \times \frac{\frac{\left[S_{1 T}-\left(S_{1 t}+\left(r-\frac{1}{2} \sigma_{1}^{2}\right) \tau\right)\right]^{2} \rho\left[S_{1 T}-\left(s_{1 t}+\left(r-\frac{1}{2} \sigma_{1}^{2}\right) \tau\right)\right]\left[S_{2 T}-\left(S_{2 t}+\left(r-\frac{1}{2} \sigma_{2}^{2}\right) \tau\right)\right]}{2 \sigma_{1}^{2} \tau}+\frac{\left[S_{2 T}-\left(S_{2 t}+\left(r-\frac{1}{2} \sigma_{2}^{2}\right) \tau\right)\right]^{2}}{2 \sigma_{1} \sigma_{2}}}{1-\rho^{2}},
\end{aligned}
$$

where: $S_{1 T}, S_{2 T}$ are the prices of the underlying assets 1 and 2 at time $T, \sigma_{1}, \sigma_{2}$ are the standard deviations of rates of return on the assets 1 and $2, r$ is the risk-free rate of return, $\rho$ is the coefficient of correlation and $\tau=T-t$.

One dimensional analogue of the equation (1) for the single asset plain vanilla European option can be expressed as:

$$
\tilde{q}\left(S_{T}\right)=\frac{1}{S_{T} \sigma \sqrt{2 \pi \tau}} \exp \left\{-\frac{\left[\ln S_{T}-\left(\ln S_{t}+\left(r-\frac{1}{2} \sigma^{2}\right) \tau\right)\right]^{2}}{2 \sigma^{2} \tau}\right\},
$$

for $0 \leq t \leq T$ and $\tau=T-t$. 
In equation (2) the natural logarithm of the market price of the underlying security is replaced by market price of the security itself.

If the price of the instrument being the basis for the analyzed contracts is equal to 60 , the risk-free rate of return amounts to $5 \%$, standard deviation of the rates of return is $20 \%$ and relative time to expiration, i.e. $t / T$ changes from 0,5 to 0,9 the distribution of the underlying asset is presented on Figure 1.

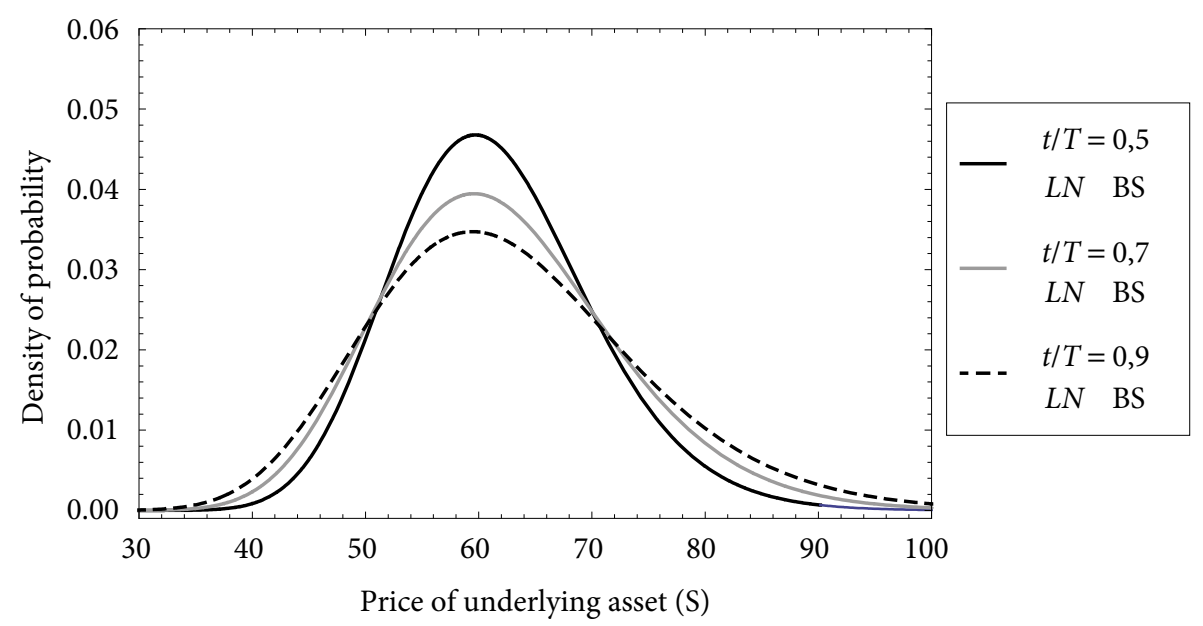

Figure 1. Distribution of underlying asset prices in the martingale method

As the BS-M method is based on the assumption that the theoretical value of the European option equals the value of future payments discounted with the risk-free rate with respect to the martingale measure $\tilde{q}$, the following formulas for the prices of the European calls and puts respectively become valid:

$$
\begin{aligned}
& C\left(S_{t}, t\right)=e^{-r \tau} E^{\tilde{q}}\left(\left(S_{T}-K, 0\right)^{+} \mid \Omega_{t}\right), \\
& P\left(S_{t}, t\right)=e^{-r \tau} E^{\tilde{q}}\left(\left(K_{T}-S_{T}, 0\right)^{+} \mid \Omega_{t}\right),
\end{aligned}
$$

where: $\tilde{q}\left(. \mid \Omega_{t}\right)$ is the probability density function dependent on filtration $\Omega_{t}$, $K$ is the strike price of the European option.

Alternatively one can write that:

$$
\begin{aligned}
& C\left(S_{t}, t\right)=e^{-r \tau} \int_{K}^{\infty}\left(S_{T}-K\right) \tilde{q}\left(S_{T} \mid \Omega_{t}\right) d S_{T}, \\
& P\left(S_{t}, t\right)=e^{-r \tau} \int_{0}^{K}\left(K-S_{T}\right) \tilde{q}\left(S_{T} \mid \Omega_{t}\right) d S_{T} .
\end{aligned}
$$


Substituting the analytical expression of probability density function for $\tilde{q}\left(S_{T} \mid \Omega_{t}\right)$ allows the formulation of the following statements:

$$
\begin{aligned}
& C\left(S_{t}, t\right)=e^{-r \tau} \int_{K}^{\infty}\left(S_{T}-K\right) \frac{1}{S_{T} \sqrt{2 \pi \sigma^{2} \tau}} e^{\left.\left.-\frac{\left\{\ln S_{T}-\left(\ln S_{t}+\left(r-\frac{1}{2} \sigma^{2}\right)\right.\right.}{2 \sigma^{2} \tau}\right)\right\}^{2}} d S_{T}, \\
& P\left(S_{t}, t\right)=e^{-r t} \int_{0}^{K}\left(K-S_{T}\right) \frac{1}{S_{T} \sqrt{2 \pi \sigma^{2} \tau}} e^{-\frac{\left\{\ln S_{T}-\left(\ln S_{t}+\left(r-\frac{1}{2} \sigma^{2}\right) \tau\right)\right\}^{2}}{2 \sigma^{2} \tau}} d S_{T} .
\end{aligned}
$$

Equations (7) and (8) can be easily extended to the two-dimensional case. In the pay-off functions of the correlation call and put options are defined respectively as (Dempster \& Hong, 2000):

$$
\begin{aligned}
& C\left(S_{1 t}, S_{2 t}, t\right)=e^{-r \tau} E^{\tilde{q}}\left(\left(S_{1 T}-K_{1}, 0\right)^{+}\left(S_{2 T}-K_{2}, 0\right)^{+} \mid \Omega_{t}\right), \\
& P\left(S_{1 t}, S_{2 t}, t\right)=e^{-r \tau} E^{\tilde{q}}\left(\left(K_{1}-S_{1 T}, 0\right)^{+}\left(K_{2}-S_{2 T}, 0\right)^{+} \mid \Omega_{t}\right),
\end{aligned}
$$

the equations allowing for the valuation of the contracts can be formulated with the use of equation (1), i.e.:

$$
\begin{aligned}
& C\left(S_{1 t}, S_{2 t}, t\right)=e^{-r \tau} \int_{K_{1} K_{2}}^{\infty} \int_{1 T}^{\infty}\left(S_{1 T}-K_{1}\right)\left(S_{2 T}-K_{2}\right) \tilde{q}\left(S_{1 T}, S_{2 T} \mid \Omega_{t}\right) d S_{2 T} d S_{1 T}, \\
& P\left(S_{1 t}, S_{2 t}, t\right)=e^{-r \tau} \int_{K_{1}}^{\infty} \int_{K_{2}}^{\infty}\left(K_{1}-S_{1 T}\right)\left(K_{2}-S_{2 T}\right) \tilde{q}\left(S_{1 T}, S_{2 T} \mid \Omega_{t}\right) d S_{2 T} d S_{1 T},
\end{aligned}
$$

where: $\tilde{q}\left(., . \mid \Omega_{t}\right)$ is the joint probability density function dependent on filtration $\Omega_{\text {t. }}$.

Unfortunately applying this procedure to the two-dimensional variable in order to conclude about the final shape of the pay-off function is highly inefficient. That is why application of an alternative approach seems to be necessary.

\section{Method of P. Carr and D. Madan}

Unlike the BS-M concept the method developed by Carr and Madan (1999) is based on the Fourier transform (the method is referred to as BS-FTCM). The approach applied to the valuation of correlation options consists of several steps. At the beginning variables representing spot prices of the underlying assets and strike prices of the option (see equations (11) and (12)) are transformed 
according to the following scheme: $s_{1 T}=\ln S_{1 T}, s_{2 T}=\ln S_{2 T}, k_{1}=\ln K_{1}, k_{2}=\ln K_{2}$. Finally, equations (13) and (14) are written as:

$$
\begin{aligned}
& C\left(s_{1 t}, s_{2 t}, t\right)=e^{-r \tau} \int_{k_{1} k_{2}}^{\infty} \int^{\infty}\left(e_{1 T}^{s_{1 T}}-e^{k_{1}}\right)\left(e^{s_{2 T}}-e^{k_{2}}\right) \tilde{q}\left(s_{1 T}, s_{2 T} \mid \Omega_{t}\right) d s_{2 T} d s_{1 T}, \\
& P\left(s_{1 t}, s_{2 t}, t\right)=e^{-r \tau} \int_{k_{1} k_{2}}^{\infty} e^{\infty}\left(e^{k_{1}}-e^{s_{1 T}}\right)\left(e^{k_{2}}-e^{s_{2 T}}\right) \tilde{q}\left(s_{1 T}, s_{2 T} \mid \Omega_{t}\right) d s_{2 T} d s_{1 T} .
\end{aligned}
$$

Limiting the analysis only to call options the equation (13) can be modified by parameters $\alpha_{1}$ and $\alpha_{2}$ in the following way:

$$
C^{m o d}\left(s_{1 t}, s_{2 t}, t\right)=e^{\alpha_{1} k_{1}+\alpha_{2} k_{2}} C\left(s_{1 t}, s_{2 t}, t\right),
$$

where: $\alpha_{1}$ and $\alpha_{2}$ are the positive constants.

Calculation of the Fourier transform of the modified function $C^{\text {mod }}\left(s_{1 t}, s_{2 t}, t\right)$ allows the conclusion that:

$$
\begin{gathered}
\psi\left(\xi_{1}, \xi_{2}\right)=\int_{-\infty}^{\infty} \int_{-\infty}^{\infty} e^{i \xi_{1} k_{1}+i \xi_{2} k_{2}} C^{\bmod }\left(s_{1 t}, s_{2 t}, t\right) d k_{2} d k_{1}= \\
=e^{-r \tau} \iint_{\mathbb{R}^{2}} \tilde{q}\left(s_{1 T}, s_{2 T} \mid \Omega_{t}\right) \frac{e^{s_{1 T}\left(1+\alpha_{1}+i \xi_{1}\right)+s_{2 T}\left(1+\alpha_{2}+i \xi_{2}\right)}}{\left(\alpha_{1}+i \xi_{1}\right)\left(\alpha_{2}+i \xi_{2}\right)\left(1+\alpha_{1}+i \xi_{1}\right)\left(1+\alpha_{2}+i \xi_{2}\right)} d \xi_{1} d \xi_{2} .
\end{gathered}
$$

Proof:

$$
\begin{gathered}
\psi\left(\xi_{1}, \xi_{2}\right)=\int_{-\infty}^{\infty} \int_{-\infty}^{\infty} e^{i \xi_{1} k_{1}+i_{2} k_{2}} C^{m o d}\left(s_{1 t}, s_{2 t}, t\right) d k_{2} d k_{1}= \\
=\int_{-\infty}^{\infty} \int_{-\infty}^{\infty} e^{i \xi_{1} k_{1}+i \xi_{2} k_{2}} e^{\alpha_{1} k_{1}+\alpha_{2} k_{2}} C\left(s_{1 t}, s_{2 t}, \tau\right) d k_{2} d k_{1}= \\
=\int_{-\infty}^{\infty} \int_{-\infty}^{\infty} e^{\xi_{1} \xi_{1}+i \xi_{2} k_{2}}\left[e^{\alpha_{1} k_{1}+\alpha_{2} k_{2}} e^{-r \tau} \int_{k_{1} k_{2}}^{\infty} \int^{\infty}\left(e^{s_{1 T}}-e^{k_{1}}\right)\left(e^{s_{2} T}-e^{k_{2}}\right)\right. \\
\left.=e^{-r \tau} \int_{-\infty}^{\infty} \int_{-\infty}^{\infty} \tilde{q}\left(s_{1 T}, s_{2 T} \mid \Omega_{t}\right) d s_{2 T} d s_{1 T}\right] d k_{2 T} d k_{1}= \\
-e^{s_{1 T}+k_{2}} e^{k_{1}\left(\alpha_{1}+i \xi_{1}\right)+k_{2}\left(\alpha_{2}+i \xi_{2}\right)}-e_{-\infty}^{s_{1}} \int^{s_{2}}\left(e^{s_{1 T}+k_{1}} e^{k_{1}} e^{k_{1}\left(\alpha_{1}+i \xi_{1}\right)+k_{2}\left(\alpha_{2}+i \xi_{2}\right)}+\right.
\end{gathered}
$$




$$
\begin{aligned}
& \left.\left.+e^{k_{1}+k_{2}} e^{k_{1}\left(\alpha_{1}+i \xi_{1}\right)+k_{2}\left(\alpha_{2}+i \xi_{2}\right)}\right) d k_{2} d k_{1}\right] d s_{2 T} d s_{1 T}= \\
& =e^{-r \tau} \int_{-\infty}^{\infty} \int_{-\infty}^{\infty} \tilde{q}\left(s_{1 T}, s_{2 T} \mid \Omega_{t}\right)\left(\frac{e^{s_{1 T}\left(1+\alpha_{1}+i \xi_{1}\right)+s_{2 T}\left(1+\alpha_{2}+i \xi_{2}\right)}}{\left(\alpha_{1}+i \xi_{1}\right)\left(\alpha_{2}+i \xi_{2}\right)}-\frac{e^{s_{1 T}\left(1+\alpha_{1}+i \xi_{1}\right)+s_{2 T}\left(1+\alpha_{2}+i \xi_{2}\right)}}{\left(\alpha_{1}+i \xi_{1}\right)\left(1+\alpha_{2}+i \xi_{2}\right)}+\right. \\
& \left.-\frac{e^{s_{1 T}\left(1+\alpha_{1}+i \xi_{1}\right)+s_{2 T}\left(1+\alpha_{2}+i \xi_{2}\right)}}{\left(1+\alpha_{1}+i \xi_{1}\right)\left(\alpha_{2}+i \xi_{2}\right)}+\frac{e^{s_{1 T}\left(1+\alpha_{1}+i \xi_{1}\right)+s_{2 T}\left(1+\alpha_{2}+i \xi_{2}\right)}}{\left(1+\alpha_{1}+i \xi_{1}\right)\left(\alpha_{2}+i \xi_{2}\right)}\right) d s_{2 T} d s_{1 T}= \\
& =e^{-r \tau} \iint_{\mathbb{R}^{2}} \tilde{q}\left(s_{1 T}, s_{2 T} \mid \Omega_{t}\right) \frac{e^{s_{1 T}\left(1+\alpha_{1}+i \xi_{1}\right)+s_{2 T}\left(1+\alpha_{2+} i \xi_{2}\right)}}{\left(\alpha_{1}+i \xi_{1}\right)\left(\alpha_{2}+i \xi_{2}\right)\left(1+\alpha_{1}+i \xi_{1}\right)\left(1+\alpha_{2}+i \xi_{2}\right)} s_{2} d s_{1} \text {. }
\end{aligned}
$$

It is worth noting that:

$$
\Phi\left(\xi_{1}, \xi_{1}\right)=\int_{-\infty}^{\infty} \int_{-\infty}^{\infty} e^{i \xi_{1} s_{1}+i \xi_{2} s_{2}} \tilde{q}\left(s_{1 T}, s_{2 T} \mid \Omega_{t}\right) d s_{2} d s_{1}
$$

is the two-dimensional characteristic function for the variables $s_{1 t}$ and $s_{2 t}$. It means that the equation (17) can be expressed as:

$$
\psi\left(\xi_{1}, \xi_{2}\right)=\frac{e^{-r \tau} \Phi\left(\xi_{1}-\left(\alpha_{1}+1\right) i, \xi_{2}-\left(\alpha_{2}+1\right) i\right)}{\left(\alpha_{1}^{2}+\alpha_{1}-\xi_{1}^{2}+i\left(2 \alpha_{1}+1\right) \xi_{1}\right)\left(\alpha_{2}^{2}+\alpha_{2}-\xi_{2}^{2}+i\left(2 \alpha_{2}+1\right) \xi_{2}\right)}
$$

Calculation of the inverse Fourier transform allows the determination of the price of the correlation call option, i.e.:

$$
\begin{gathered}
C\left(s_{1 t}, s_{2 t}, t\right)=\frac{e^{-\alpha_{1} k_{1}-\alpha_{2} k_{2}}}{2 \pi} \int_{-\infty}^{\infty} \int_{-\infty}^{\infty} e^{-i \xi_{1} k_{1}-i \xi_{2} k_{2}} \psi\left(\xi_{1}, \xi_{2}\right) d \xi_{2} d \xi_{1}=\frac{e^{-\alpha_{1} k_{1}-\alpha_{2} k_{2}}}{2 \pi} \times \\
\times \int_{-\infty}^{\infty} \int_{-\infty}^{\infty} e^{-i \xi_{1} k_{1}-i \xi_{2} k_{2}} \frac{e^{-r \tau} \Phi\left(\xi_{1}-\left(\alpha_{1}+1\right) i, \xi_{2}-\left(\alpha_{2}+1\right) i\right)}{\left(\alpha_{1}^{2}+\alpha_{1}-\xi_{1}^{2}+i\left(2 \alpha_{1}+1\right) \xi_{1}\right)\left(\alpha_{2}^{2}+\alpha_{2}-\xi_{2}^{2}+i\left(2 \alpha_{2}+1\right) \xi_{2}\right)} d \xi_{2} d \xi_{1} .
\end{gathered}
$$

If the prices of the instruments being the basis for the analyzed contracts are equal to 60 and 62, risk-free rate of return amounts to 5\%, standard deviations of the rates of return are $10 \%$ and $5 \%$, relative time to expiration is equal to 0,1 , coefficient of correlation $\rho=0$ and $\alpha_{1}=\alpha_{2}=1$ the payoff function of the correlation call option is presented on Figure 2.

Although the BS-FTCM method can be applied to pricing correlation options it is far from being perfect. The biggest problem that appears when using the method is associated with the tendency of the subintegral function from 


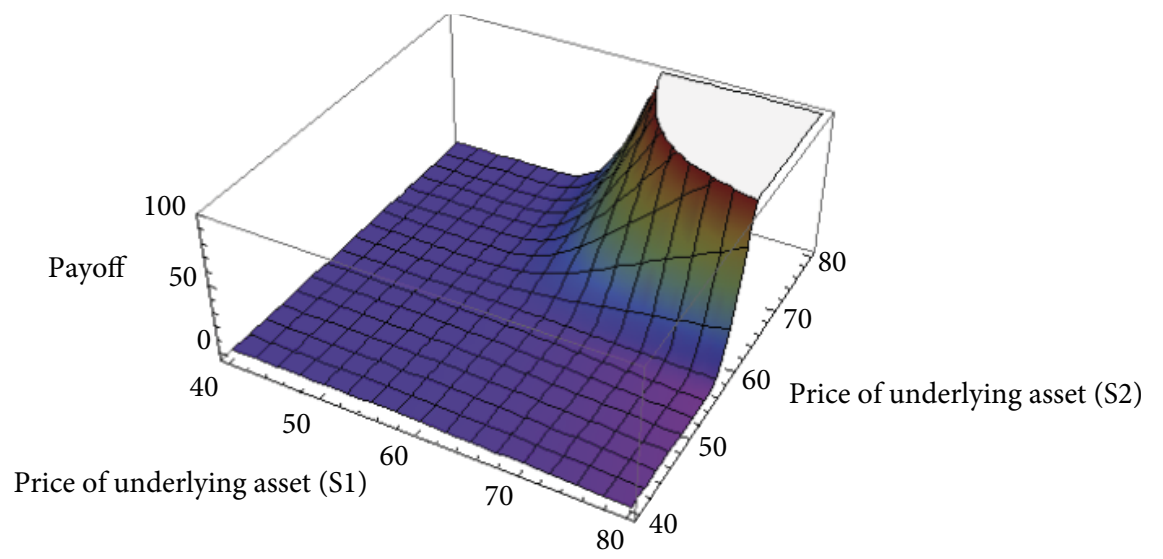

Figure 2. Pay-off function of the correlation call option in the BS-FTCM method

equation (20) to rapid oscillation when out-of-the-money (OTM) near-to-expiration contracts are considered. That is why other concepts for pricing options should be explored.

\section{Alternative methods of pricing options utilizing Fourier transform}

There are many alternative concepts based on the Fourier transform which can be used to value derivatives (Orzechowski, 2016). To the best knowledge of the author only two of them were applied to pricing correlation options. Nevertheless almost all of them can be good alternatives to the BS-M and BSFTCM methods. Only the four most important approaches are presented below.

The first method of pricing options using Fourier transform was developed by Bakshi and Madan (the method is referred to as BS-FTBM) (Bakshi \& Madan, 2000). In this approach the price of the underlying assets and the exercise price of the option in equation (5) are converted to logarithmic values. Then the right hand side of a new equation is split into two parts. For each of them Fourier transforms are determined and reversed. Finally the theoretical value of the option may be obtained with the use of equation (21):

$C\left(S_{t}, t\right)=\frac{1}{2}\left(S_{t}-K e^{-r \tau}\right)+\frac{1}{\pi} \int_{0}^{\infty} S_{t^{\prime}} \mathfrak{R}\left[\frac{e^{-i \xi k} \phi(\xi-i)}{i \xi \phi(-1)}\right] d \xi-K e^{-r \tau} \frac{1}{\pi} \int_{0}^{\infty} \mathfrak{R}\left[\frac{e^{-i \xi k} \phi(\xi)}{i \xi}\right] d \xi$

where: $\mathfrak{R}$ is the real part of the subintegral function and $i$ is the imaginary part of the complex number. 
The second method of pricing options with the use of the Fourier transform was derived by Attari (the method is referred to as BS-FTA) (Attari, 2004). This approach begins with the generalization of the process responsible for the stock price movements. Next equation (5) is transformed into the following formula:

$$
S_{t} \int_{l}^{\infty} e^{x(t, T)} \tilde{q}(x(t, T)) d x(t, T)-e^{-r \tau} K \int_{l}^{\infty} \tilde{q}(x(t, T)) d x(t, T)=S_{t} \pi_{1}-e^{-r \tau} K \pi_{2},
$$

where: $l=K / S_{t} e^{r \tau}$ and $x(t, T)$ is the unpredictable shock in prices of the security.

Noting that $0 \leq \int_{l}^{\infty} e^{x(t, T)} \tilde{q}(x(t, T)) d x(t, T) \leq 1$ allows to treat both $\pi_{1}$ and $\pi_{2}$ as probability density functions. Determining their Fourier and inverse Fourier transforms leads directly to the price of the option, i.e.:

$$
\begin{gathered}
C\left(S_{t}, t\right)=S_{t}\left(1+\frac{e^{l}}{\pi} \int_{0}^{\infty} \mathfrak{R}\left[\phi(\xi)\left(\frac{e^{-i \xi l}}{i(\xi+i)}\right)\right] d \xi\right)+ \\
-e^{-r(T-t)} K\left(\frac{1}{2}+\frac{1}{\pi} \int_{0}^{\infty} \Re\left[\phi(\xi)\left(\frac{e^{-i \xi l}}{i \xi}\right)\right] d \xi\right) .
\end{gathered}
$$

The third method of pricing option using Fourier transform was proposed by Bates (the method is referred to as BS-FTB) (Bates, 2006). This approach is based on the modification of the previous concept. As before equation (5) is transformed but in this case to the following form:

$$
C\left(S_{t}, t\right)=S_{0}-e^{-r \tau} K \int_{-\infty}^{K} \frac{S_{T}}{K} \tilde{q}\left(S_{T} \mid \Omega_{t}\right) d S_{T}-e^{-r \tau} \int_{K}^{\infty} K \tilde{q}\left(S_{T} \mid \Omega_{t}\right) d S_{T} .
$$

Next, it is modified and Fourier transformed. Finally, the price of the option is determined in the following way:

$$
C\left(S_{t}, t\right)=S_{0}-e^{-r \tau} K\left(\frac{1}{2}+\frac{1}{\pi} \int_{0}^{\infty} \Re\left[\frac{e^{-i \xi k}}{i \xi(1-i \xi)} \phi(\xi)\right] d \xi\right) .
$$

The fourth method of pricing options using Fourier transform was developed by Lewis (the method is referred to as BS-FTL) (Lewis, 2001). This concept is based on a multiple transformation of the equation (5), conversion of the dependent variables to logarithmic values and calculation of the Fourier and inverse Fourier transforms. Finally, the price of the option can be determined with the use of the following formula: 


$$
C\left(S_{t}, t\right)=S_{t}-\frac{\sqrt{S_{t} K} e^{-\frac{r \tau}{2}}}{\pi} \int_{0}^{\infty} \mathfrak{R}\left[\phi\left(u-\frac{i}{2}\right) \frac{e^{i u k}}{u^{2}+\frac{1}{4}}\right] d u .
$$

Although all the methods presented above can be easily applied to the valuation of correlation options (Fan \& Wang, 2017), the remaining part of the article is devoted only to the new method of pricing options based on the Fourier transform and its application to the valuation of correlation options.

\section{New method of pricing correlation options using Fourier transform}

The new method of pricing options using Fourier transform (the method is referred to as BS-FTAu) consists of several steps. At the beginning dependent variables in equation (5) are converted to logarithmic values. Next, the right hand side of the equation obtained is split into two parts, i.e.:

$$
C\left(S_{t}, t\right)=e^{-r \tau} \int_{k}^{\infty} e^{s_{T}} \tilde{q}\left(s_{T} \mid \Omega_{t}\right) d s_{T}-e^{-r \tau} \int_{k}^{\infty} e^{k} \tilde{q}\left(s_{T} \mid \Omega_{t}\right) d s_{T^{*}}
$$

The first part of equation (27) is Fourier transformed as in BS-FTBM model, i.e.:

$$
\Psi_{T}^{1}(\xi)=\int_{-\infty}^{\infty} e^{i \xi k}\left[e^{-r \tau} \frac{\int_{k}^{\infty} e^{s_{T}} \tilde{q}\left(s_{T} \mid \Omega_{t}\right) d s_{T}}{\int_{-\infty}^{\infty} e^{s_{T}} \tilde{q}\left(s_{T} \mid \Omega_{t}\right) d s_{T}} \int_{-\infty}^{s_{T}} \tilde{q}\left(s_{T} \mid \Omega_{t}\right) d s_{T}\right] d k .
$$

It can easily be seen that $\int_{-\infty}^{\infty} e^{s_{T}} \tilde{q}\left(s_{T} \mid \Omega_{t}\right) d s_{T}$ may be treated both as the characteristic function of $s_{T}$ assuming $\xi=-i$, i.e. $\phi(-i)$, and expected value of $S_{T}$. It allows the transformation of the equation (28) to the following form:

$$
\Psi_{T}^{1}(\xi)=\frac{S_{0} \phi(\xi-i)}{i \xi \phi(-i)}
$$


Proof:

$$
\begin{gathered}
\Psi_{T}^{1}(\xi)=\int_{-\infty}^{\infty} e^{i \xi k}\left[e^{-r T} \frac{\int_{k}^{\infty} e^{s_{T}} \tilde{q}\left(s_{T} \mid \Omega_{t}\right) d s_{T}}{\phi(-i)} S_{0} e^{r T}\right] d k=S_{0} \int_{-\infty}^{\infty} e^{i \xi k}\left[\frac{\int_{k}^{\infty} e^{s_{T}} \tilde{q}\left(s_{T} \mid \Omega_{t}\right) d s_{T}}{\phi(-i)}\right] d k= \\
=\frac{S_{0}}{\phi(-i)} \int_{-\infty}^{\infty} e^{i \xi k}\left[\int_{k}^{\infty} e^{s_{T}} \tilde{q}\left(s_{T} \mid \Omega_{t}\right) d s_{T}\right] d k=\frac{S_{0}}{\phi(-i)} \int_{-\infty}^{\infty} \tilde{q}\left(s_{T} \mid \Omega_{t}\right)\left[\int_{-\infty}^{s_{T}} e^{i \xi k} e^{s_{T}} d k\right] d s_{T}= \\
=\frac{S_{0}}{\phi(-i)} \int_{-\infty}^{\infty} \tilde{q}\left(s_{T} \mid \Omega_{t}\right)\left[\frac{e^{i \xi k+s_{T}}}{i \xi}\right]_{-\infty}^{s_{T}} d s_{T}= \\
=\frac{S_{0}}{\phi(-i)} \int_{-\infty}^{\infty} \tilde{q}\left(s_{T} \mid \Omega_{t}\right)\left[\frac{e^{s_{T}(1+i \xi)}}{i \xi}\right] d s_{T}=\frac{S_{0} \phi(\xi-i)}{i \xi \phi(-i)} .
\end{gathered}
$$

The second part of equation (27) is also Fourier transformed, i.e.:

$$
\Psi_{T}^{2}(\xi)=\int_{-\infty}^{\infty} e^{i \xi k} \int_{k}^{\infty} e^{k} \tilde{q}\left(s_{T} \mid \Omega_{t}\right) d s_{T} d k=\frac{\phi(\xi-i)}{i \xi+1} .
$$

Proof:

$$
\begin{gathered}
\Psi_{T}^{2}(\xi)=K \int_{-\infty}^{\infty} e^{i \xi k}\left[e^{-r T} \int_{k}^{\infty} \tilde{q}\left(s_{T} \mid \Omega_{t}\right) d s_{T}\right] d k= \\
=K e^{-r T} \int_{-\infty}^{\infty} \tilde{q}\left(s_{T} \mid \Omega_{t}\right)\left[\int_{-\infty}^{s_{T}} e^{i \xi k} d k\right] d s_{T}= \\
=K e^{-r T} \int_{-\infty}^{\infty} \tilde{q}\left(s_{T} \mid \Omega_{t}\right)\left[\frac{e^{i \xi k}}{i \xi}\right]_{-\infty}^{s_{T}} d s_{T}= \\
=K e^{-r T} \int_{-\infty}^{\infty} \tilde{q}\left(s_{T} \mid \Omega_{t}\right) \frac{e^{i \xi \xi_{T}}}{i \xi} d s_{T}=K e^{-r T} \frac{\phi(\xi)}{i \xi} .
\end{gathered}
$$

Inverting both Fourier transforms allows obtention of the final price of the option, i.e.:

$$
C\left(S_{t}, t\right)=\frac{1}{2} S_{t}-e^{-r \tau} \frac{1}{\pi} \int_{0}^{\infty} \mathfrak{R}\left[e^{-i \xi k} \frac{\phi(\xi-i)}{i \xi(i \xi+1)}\right] d \xi .
$$

Before applying equation (33) to the valuation of correlation options it pays to transform equation (13) to the form: 


$$
C\left(s_{1 t}, s_{2 t}, t\right)=e^{-r \tau} \int_{k_{1} k_{2}}^{\infty}\left(e^{s_{1 T}}-e^{k_{1}}\right)\left(e^{s_{2 T}}-e^{k_{2}}\right) \tilde{q}\left(s_{2 T} \mid s_{1 T}\right) \tilde{q}\left(s_{1 T}\right) d s_{2 T} d s_{1 T},
$$

where: $\tilde{q}\left(s_{2 T} \mid s_{1 T}\right)$ is the conditional probability density function of $s_{2 T}$ given $s_{1 T}$ and $\tilde{q}\left(s_{1 T}\right)$ is the probability density function of $s_{1 T}$ (Carmona \& Durrleman, 2003).

It is worthy of note that $s_{1 T}$ and $s_{2 T}$ are normally distributed variables with known analytical probability distribution functions.

As the application of the formula (33) to equation (34) is evident the closedform formula for the final price of the correlation option is presented without derivation (Feunou \& Tafolong, 2015).

$$
\begin{aligned}
C\left(s_{1 t}, s_{2 t}, t\right) & =e^{r \tau}\left(\frac{1}{2} S_{1 t}-e^{-r \tau} K_{1}\left(\frac{1}{2}-\frac{1}{\pi} \int_{0}^{\infty} \Re\left[e^{-i \xi_{1} k_{1}} \frac{\varphi\left(\xi_{1}-i\right)}{i \xi_{1}\left(i \xi_{1}+1\right)}\right] d \xi_{1}\right)\right) \\
& \left(\frac{1}{2} S_{2 t}-e^{-r \tau} \frac{1}{\pi} \int_{0}^{\infty} \Re\left[e^{-i \xi_{2} k_{2}} \frac{\varphi\left(\xi_{2}-i\right)}{i \xi_{2}\left(i \xi_{2}+1\right)}\right] d \xi_{2}\right) .
\end{aligned}
$$

It should be noted that equation (35) is correct but only under the previously introduced assumption that $\rho=0$.

Given the same data as previously the pay-off function of the correlation option in the BS-FTAu method can be easily determined (see Figure 3).

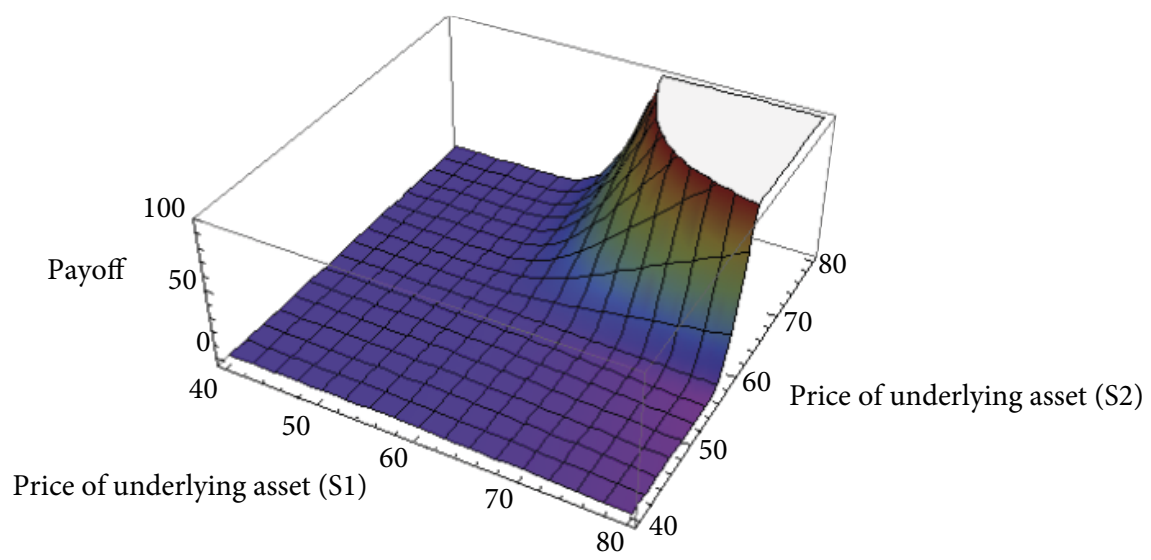

Figure 3. Pay-off function of the correlation call option in the BS-FTAu method

Applying the BS-FTAu method for the calculation of the correlation option has one advantage over other concepts based on the Fourier transform. The speed of calculating the final price of the option is greater than in other approaches. 
It is worth noting that the presented method can be easily applied to other models of pricing options, e.g. jump-diffusion models (Kou, 2002; Merton, 1976), pure jump models (Carr, Geman, Madan, \& Yor, 2002; Kirkby, 2017; Madan, Carr, \& Chang, 1998). The biggest benefits resulting from the application of the Fourier transform and the BS-FTAu method to the pricing of options, however, appear in stochastic volatility models (Heston, 1993; Hull \& White, 1987; Stein \& Stein, 1991).

\section{Conclusions}

Pricing derivatives is an important issue in the financial industry. For that reason efficiently functioning methods allowing for the valuation of the contracts constructed on the basis of laws whose execution may be demanded by one party from the other attracts the particular attention of many researchers.

In this article several methods of pricing options based on the Fourier transform were presented and two of them were applied to the valuation of correlation options. The first approach, i.e. the one proposed by Carr and Madan (1999) seems to be inefficient due to the fact that the subintegral function in equation (20) becomes highly oscillatory, especially for the OTM near-to expiration options. In consequence, alternative methods have to be explored. Although many concepts in this field were developed, only one, proposed by the author, was fully derived in the article. The method allows the pricing of the correlation options more efficiently. The main reasons for the superiority of the method over other approaches are: a more efficient scheme of calculating the Fourier transform and replacing the joint probability density function with the two other probability density functions. In consequence a new and better method based on the Fourier transform which can be applied to the valuation of the correlation options is proposed.

\section{References}

Attari, M. (2004). Option pricing using Fourier transforms: A numerically efficient simplification. Retrieved from http://papers.ssrn.com/sol3/papers.cfm?abstract_ id $=520042$. doi: $10.2139 /$ ssrn. 520042

Bakshi, G., \& Madan, D. (2000). Spanning and derivative - security valuation. Journal of Financial Economics, 55(2), 205-238. doi: 10.1016/S0304-405X(99)00050-1

Bates, D. (2006). Maximum likelihood estimation of latent affine processes. Review of Financial Studies, 19(3), 909-965. doi: 10.1093/rfs/hh

Black, F., \& Scholes, M. (1973). The pricing of options and corporate liabilities. The Journal of Political Economy, 81(3), 637-654. doi: 10.1086/260062 
Carmona, R., \& Durrleman, V. (2003). Pricing and hedging spread options. SIAM Review, 45(4), 627-285. doi: 10.1137/S0036144503424798

Carr, P., Geman, H., Madan, D., \& Yor, M. (2002). The fine structure of asset returns: An empirical investigation. Journal of Business, 75(2), 305-332. doi: 10.1086/338705

Carr, P., \& Madan, D. (1999). Option valuation using the fast Fourier transform. Journal of Computational Finance, 2(4), 61-73. doi: 10.21314/JCF.1999.043

Dempster, M. A. H., \& Hong, S. S. G. (2000). Spread option valuation and fast Fourier transform. Retrieved from http://citeseerx.ist.psu.edu/viewdoc/download?doi=10 .1.1.27.9431\&rep=rep1\&type=pdf. doi: 10.1007/978-3-662-12429-1_10

Fan, K., \& Wang, R. (2017). Valuation of correlation options under a stochastic interest rate model with regime switching. Frontiers of Mathematics in China, 12(5), 1113-1130. doi: 10.1007/s11464-017-0608-5

Feunou, B., \& Tafolong, E. (2015). Fourier inversion formulas for multiple-asset option pricing. Studies in Nonlinear Dynamics \& Econometrics, 19(5), 531-559. doi: 10.1515/snde-2014-0034

Heston, S. L. (1993). A closed form solution for options with stochastic volatility with applications to bond and currency options. Review of Financial Studies, 6(2), 327-343. doi: 10.1093/rfs/6.2.327

Hull, J., \& White, A. (1987). The pricing of options on assets with stochastic volatilities. Journal of Finance, 42(2), 281-300. doi: 10.1111/j.1540-6261.1987.tb02568.

Kirkby, J. L. (2017). Robust barrier option pricing by frame projection under exponential Lévy dynamics. Applied Mathematical Finance, 24(4), 337-386. doi: 10.1080/1350486X.2017.1384701

Kou, S. G. (2002). A jump-diffusion model for option pricing. Management Science, 48(8), 1086-1101. doi: 10.2139/ssrn.242367

Lewis, A. (2001), A simple option formula for general jump-diffusion and other exponential levy processes. Retrieved from http://papers.ssrn.com/sol3/papers. cfm?abstract_id=282110. doi: 10.2139/ssrn.282110

Madan, D. B., Carr, P., \& Chang, E. C. (1998). The variance gamma process and option pricing. European Finance Review, 2, 79-105. doi: 10.1023/A:1009703431535

Merton, R. C. (1976). Option pricing when underlying stock returns are discontinuous. Journal of Financial Economics, 3(1-2), 125-144. doi: 10.1016/0304-405X(76)90022-2

Orzechowski, A. (2016). Analiza efektywności obliczeniowej opcji na przykładzie modelu F. Blacka i M. Scholesa. Finanse, 9(1), 137-154.

Stein, E., \& Stein, J. (1991). Stock price distributions with stochastic volatility. Review of Financial Studies, 4(4), 727-752. doi: 10.1093/rfs/4.4.727

Zhu, J. (2000). Modular pricing of options. An application of Fourier analysis. Heidelberg: Springer-Verlag. doi: 10.1007/978-3-662-04309-7 